\title{
Update on the association between dry eye disease and meibomian gland dysfunction
}

\author{
Tommy CY Chan *, Sharon SW Chow, Kelvin HN Wan, Hunter KL Yuen
}

\section{A B S T R A C T}

Dry eye disease is one of the most common ophthalmic complaints; it results from the activity of various pathways and is considered a multifactorial disease. An important factor that contributes to the onset of dry eye disease is meibomian gland dysfunction. Meibomian gland dysfunction causes a disruption in the tear film lipid layer which affects the rate of tear evaporation. This evaporation leads to tear hyperosmolarity, eventually triggering the onset of dry eye disease. Dry eye disease and meibomian gland dysfunction are strongly associated with each other, such that many of their risk factors, signs, and symptoms overlap. This review aimed to provide an update on the association between dry eye disease and meibomian gland dysfunction. A stepwise approach for diagnosis and management is summarised.
Hong Kong Med J 2019;25:38-47

https://doi.org/10.12809/hkmj187331

1,2,3 TCY Chan *, MB, BS, MMedSc

${ }_{3,4}$ SSW Chow, MB, BS

${ }^{1,5}$ KHN Wan, MB, BS

${ }_{1,6} \mathrm{HKL}$ Yuen, MB, ChB

1 Department of Ophthalmology and Visual Sciences, The Chinese University of Hong Kong, Shatin, Hong Kong

Hong Kong Sanatorium \& Hospital, Happy Valley, Hong Kong

Department of Ophthalmology, The University of Hong Kong, Cyberport, Hong Kong

${ }^{4}$ Department of Ophthalmology, Grantham Hospital, Wong Chuk Hang, Hong Kong

${ }^{5}$ Department of Ophthalmology, Tuen Mun Hospital, Tuen Mun, Hong Kong

Hong Kong Eye Hospital, Hong Kong

* Corresponding author: tommychan.me@gmail.com

\section{Introduction}

Dry eye disease (DED) is one of the most common ocular surface diseases, which can significantly affect the quality of life of affected patients. The definition of DED has been progressively established in recent decades. The goal of the Tear Film and Ocular Surface Society (TFOS) Dry Eye Workshop (DEWS) is to create an evidence-based definition, a well-defined classification system, and an appropriate diagnosis and management algorithm for DED. ${ }^{1}$ In 2007, the TFOS DEWS definition of DED was first published. ${ }^{2}$ In 2017, the TFOS DEWS II amended the definition of DED to be 'a multifactorial disease of the ocular surface, characterised by a loss of homeostasis of the tear film, and accompanied by ocular symptoms, in which tear film instability and hyperosmolarity, ocular surface inflammation and damage, and neurosensory abnormalities play etiological roles.' The term 'multifactorial' indicates that the disease occurs as a result of multiple influential factors, while the term 'etiological roles' suggests the involvement of various pathways in the onset of DED. ${ }^{1}$ In 2017, the Asia Dry Eye Society also agreed upon a new definition of DED, as 'a multifactorial disease characterised by unstable tear film causing a variety of symptoms and or visual impairment, potentially accompanied by ocular surface damage. ${ }^{3}$

The two main categories of DED are evaporative dry eye and aqueous deficient dry eye. ${ }^{2}$ Evaporative dry eye is related to conditions that affect the eyelids, such as meibomian gland dysfunction (MGD), poor blinking effort, and lid disorders, or that affect the ocular surface, such as prolonged contact lens wear, frequent use of topical drug preservatives, and immune-related ocular surface disorders (eg, atopic keratoconjunctivitis). Aqueous deficient dry eye is primarily due to conditions affecting lacrimal gland function, such as Sjögren's syndrome, lacrimal gland duct obstruction or deficiencies, and adverse effects of systemic drugs. Epidemiological evidence suggests that DED is mainly evaporative in nature, ${ }^{4}$ and is often associated with MGD. ${ }^{5,6}$

Meibomian glands are found in the upper and lower eyelids, where they secrete lipids (meibum) onto the ocular surface, forming the outermost layer of the tear film. These lipids spread easily, promoting tear film stability and protecting against evaporation. Meibomian gland dysfunction is defined as 'a chronic, diffuse abnormality of the meibomian glands, commonly characterised by terminal duct obstruction and/or qualitative/quantitative changes in the glandular secretion. It may result in alteration of the tear film, symptoms of eye irritation, clinically apparent inflammation and ocular surface disease.?

This review aims to provide an update on the association between MGD and DED, with particular attention to the diagnosis and management of these conditions. We will discuss the epidemiology, 
pathophysiology, risk factors, signs and symptoms, diagnosis, and ancillary imaging of MGD and DED, along with appropriate behaviour, medical, and surgical management.

\section{Methods}

A comprehensive literature search on PubMed was performed for studies published between January 2006 and December 2017 with keywords 'dry eye', 'dry eye disease', 'tear film,' 'meibomian gland', and 'meibomian gland dysfunction'. Search results were limited to clinical studies published in English. Articles reporting DED and MGD were reviewed. Particular emphasis was placed on papers that investigated the association between DED and MGD. The reference lists of the retrieved articles were also examined for relevant studies.

\section{Epidemiology}

The reported prevalence of DED ranges from $5 \%$ to $50 \%,{ }^{4}$ whereas the reported prevalence of MGD varies more widely from $3.5 \%$ to nearly $70 \% .{ }^{8,9}$ Meibomian gland dysfunction appears to be more prevalent in Asian populations..$^{5}$ Meibomian gland dysfunction has been reported to contribute to $60 \%$ of all cases of DED; an additional 20\% of cases of DED are caused by aqueous deficiency.

\section{Pathophysiology of dry eye disease}

All forms of DED primarily occur because of water loss from the tear film, which leads to tear hyperosmolarity due to evaporative dry eye and/or aqueous deficient dry eye. ${ }^{10}$ In evaporative dry eye, hyperosmolarity results from excessive evaporation of tears in the context of normal lacrimal function. In contrast, in aqueous deficient dry eye, hyperosmolarity occurs due to an inadequate rate of lacrimal secretion in the context of a normal rate of evaporation. Environmental factors affect the presence of hyperosmolarity on the ocular surface, which may trigger the onset of DED, or cause worsening of the condition.

\section{Pathophysiology of meibomian gland dysfunction}

Meibomian gland dysfunction is classified according to the rate of gland secretion. A low delivery state is characterised by meibomian gland hyposecretion or obstruction, whereas a high delivery state is characterised by meibomian gland hypersecretion. Of these two categories, the most common mechanism is a low delivery state due to duct obstruction. ${ }^{11}$ Epithelial hyperkeratinisation is the most common cause of duct obstruction, leading to meibum accumulation with chronic inflammation and, eventually, gland dropout. ${ }^{12}$ Importantly, this results

\section{眼症與瞼板腺功能障礙關係的最新進展} 陳頌恩、㱀樞韻、尹浩柍、袁國禮

乾眼症是最常見的眼科疾病之一。它可由各種途徑的活動引起, 被認 為是一種多因素疾病。導致乾眼症發病的其一重要因素為瞼板腺功能 障礙。瞼板腺功能障礙導致淚膜脂質層受破壞, 影響淚液蒸發速率。 這種蒸發導致淚液高滲透壓, 最終引發乾眼症。乾眼症和瞼板腺功能 障礙密切相關, 因此不少風險因素、體徵和症狀均相互重疊。本文提 供乾眼症與瞼板腺功能障礙間關聯的最新進展, 並總結逐步式診斷和 管理的方法

in the quantitative and qualitative abnormalities of glandular secretions. There is a high prevalence of MGD in acne rosacea, which is a chronic cutaneous inflammatory disorder.

\section{Association between dry eye disease and meibomian gland dysfunction}

The tear film consists of three distinct layers: the lipid, aqueous, and mucus layers. The lipid layer, a key component of the tear film, is derived from meibomian glands. The lipid layer prevents water evaporation from the ocular surface and is thus crucial in the maintenance of a healthy ocular surface. Dysfunction of the meibomian glands results in unbalanced lipid secretion, thereby increasing the rate of ocular surface evaporation and causing tear hyperosmolarity. ${ }^{13}$ Patients with MGD reportedly exhibit a higher tear evaporation rate than that of normal subjects. ${ }^{13}$ This shows that DED is directly correlated with the integrity and quality of meibum on the ocular surface.

\section{Risk factors}

Many risk factors associated with DED also contribute to MGD. Thus, risk factor modifications can likely improve both disease states.

\section{Sex}

Female sex is a significant risk factor for the development of both DED and MGD., ${ }^{5,6}$ This may be due to the effect of hormonal changes on meibomian secretion, as androgen and oestrogen receptors are both present within the meibomian glands. $^{14}$ Importantly, androgens have been reported to stimulate meibum secretion and suppress inflammation, whereas oestrogens reduce meibum secretion and increase inflammation. ${ }^{15}$ Dysfunctional meibomian gland secretion and concurrent alterations in the lipid layer have been 
observed in patients with androgen depletion. ${ }^{16}$ Additionally, female sex has been identified as a risk factor for the development of autoimmune diseases that lead to DED, such as Sjögren's syndrome. ${ }^{17}$

\section{Topical medications}

Topical medications can cause both DED and MGD; this may be a result of ocular surface disturbances with various aetiologies, including allergic reactions, toxic epitheliopathy, and inflammatory response from chronic chemical irritation. Multiple studies have revealed a clear relationship between the prevalence of dry eye and increasing use of eye drops. ${ }^{18}$ The primary factor underlying this relationship is the presence of benzalkonium chloride preservative agent in topical medications. Benzalkonium chloride has been strongly linked with the onset of DED, as it dissolves the lipid tear film layer and has been shown to disrupt tear film osmolarity. ${ }^{19}$ Similarly, DED and MGD are commonly reported in glaucoma patients who use topical glaucoma medications, which contain benzalkonium chloride. Use of these medications has been associated with changes in meibomian gland structure, leading to MGD. ${ }^{20}$

\section{Contact lens wear}

Contact lens wear is commonly associated with the onset of both DED and MGD. An epidemiological study showed that $50 \%$ of contact lens wearers experience dry eye symptoms, whereas only $22 \%$ of non-contact lens wearers experience such symptoms. ${ }^{21}$ Contact lens wear alters the integrity of the tear film: a thinner lipid layer has been observed in contact lens wearers, which causes an increased tear evaporation rate and tear hyperosmolarity..$^{22}$ Environmental factors, such as prolonged usage of visual display devices, as well as air pollution and seasonal changes, further aggravate dry eye symptoms in contact lens wearers. The occurrence of MGD in contact lens wearers is suspected to be a result of chronic inflammation, ${ }^{23}$ as well as clogging of gland orifices due to accumulation of desquamated epithelial cells. ${ }^{24}$ Contact lens wearers demonstrate a high percentage of meibomian gland dropout and reduction in gland function; these aspects are reportedly directly related to the duration of contact lens wear. ${ }^{25}$

\section{Refractive surgery}

Worldwide, laser in situ keratomileusis (LASIK) is the most common corneal refractive surgery currently in use. Dry eye disease is often associated with a history of LASIK, and can be aggravated by both preoperative and postoperative factors. Preoperatively, the risk of DED is significantly increased in patients who are long-term contact lens wearers, as well as in patients whose eyes exhibit pre-existing tear film instability. ${ }^{26}$ Greater refractive correction magnitude requires deeper ablation, resulting in a greater extent of sensory nerve damage. This nerve damage results in reduced corneal sensitivity, leading to neuropathic dry eyes. Notably, this mechanism is the most common aetiology of post-LASIK dry eyes. ${ }^{26}$ Corneal refractive surgery has also been shown to reduce corneal epithelial integrity, conjunctival goblet cell concentration, and meibomian gland function, resulting in lower ocular surface disease index and ocular surface staining scores. ${ }^{27}$

\section{Demodicosis}

Two species of mites, Demodex folliculorum and Demodex brevis, are the only mites that affect human skin; such infestations are known as demodicosis. ${ }^{28}$ Reportedly, $D$ folliculorum infests the lash follicles, whereas $D$ brevis infests the meibomian glands. ${ }^{28}$ These infestations increase the meibum melting temperature, resulting in a more viscous lipid layer. A recent study showed that a higher $D$ brevis count was associated with more severe MGD. ${ }^{29}$ Furthermore, confocal microscopy analysis revealed lower counts of Demodex mites in the glands of healthy subjects than in the glands of patients with MGD-related DED. ${ }^{30}$ The role of Demodex mites in the pathology of MGD has not been fully elucidated; however, eradication of Demodex is particularly helpful in relieving related ocular symptoms. Thus, there may a pathogenic role for Demodex infestation in MGD.

\section{Symptoms}

Many signs and symptoms of DED overlap with those of MGD. However, most patients with MGD are largely or entirely asymptomatic; if they are symptomatic, their particular symptoms often do not directly correlate with the severity of ocular surface disturbance. In a population-based study in China, $22 \%$ of the study population demonstrated asymptomatic MGD, while $9 \%$ showed symptomatic MGD. ${ }^{8}$ In cases of symptomatic MGD, patients report a variety of symptoms, including foreign body sensation, dryness, itching, and/or photosensitivity. ${ }^{7}$ These manifestations may be linked to chronic inflammation or mechanical friction between the ocular surface and meibum that has accumulated in the gland orifices.

\section{Ocular surface signs and diagnosis}

Because DED and MGD are common ophthalmic problems, a clear diagnosis is crucial for suitable management. Appropriate tests should be used to diagnose and monitor DED, in accordance with the revised TFOS DEWS II definition of the disease. For these purposes, the TFOS DEWS II proposed a battery of diagnostic tests for DED. 


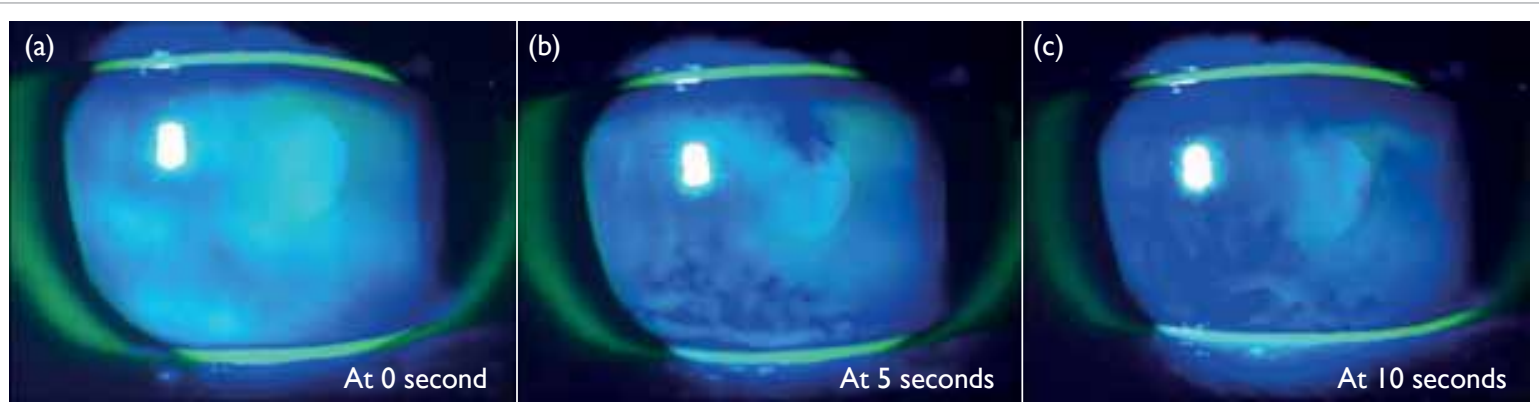

FIG I. Demonstration of tear breakup time in a patient with dry eye disease.After instillation of fluorescein staining in the eye, the patient was asked to maintain the eye open without blinking. Tear breakup time is defined as the duration from the beginning of eye open (a) to the first appearance of black spots on the corneal surface (b and c)

The diagnostic tests begin with triaging questions and risk factor analysis. These are followed by screening for symptoms using standardised questionnaires, including the five-item dry eye questionnaire or the ocular surface disease index. Markers of homeostasis used in diagnostic testing include measures of tear breakup time, staining of the ocular surface, Schirmer's test, and tear osmolarity. Tear breakup time is a non-invasive measurement that is defined as the time required for the tear film to break up sufficiently that the patient can no longer refrain from blinking. ${ }^{31}$ A tear breakup time of $<10$ seconds is considered diagnostic for DED (Fig 1). Ocular surface staining is performed by fluorescein staining for corneal damage and lissamine green staining for conjunctival and lid margin damage (Fig 2). ${ }^{31}$ Schirmer's test consists of the placement of a small strip of filter paper inside the lower fornix with the eye closed. After 5 minutes, the amount of moisture is measured as the distance that tear moisture has travelled on the paper, due to capillary action; a value of $<5 \mathrm{~mm}$ indicates DED. Finally, tear osmolarity should be assessed with a calibrated device; a positive result is defined as $\geq 308 \mathrm{mOsm} / \mathrm{L}$ in the measured eye, or a difference of $>8 \mathrm{mOsm} / \mathrm{L}$ between two eyes. ${ }^{32}$

The Asia Dry Eye Society recommends diagnosis of DED by using a combination of symptoms assessed by standardised questionnaires (ocular surface disease index, McMonnies questionnaire, women's health study questionnaire, or five-item dry eye questionnaire), together with a reduced tear breakup time (with a cut-off value of $<5 \mathrm{~s}$ ). ${ }^{3}$

Clinical diagnosis of MGD is made based on the examination of altered anatomical features, such as meibomian gland dropout, altered meibum excretion, and changes to lid morphology, with plugging or pouting of the gland orifice. Meibomian glands with normal appearance are shown in Figure 3. Gentle gland expression with digital pressure to the central lower lid can evaluate terminal duct obstruction and meibum quality (Fig 4). Subtype

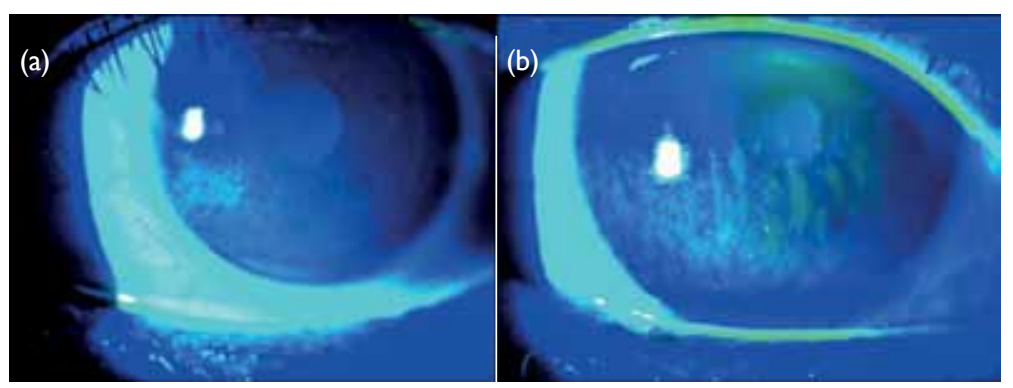

FIG 2. Corneal fluorescein staining pattern in patients with (a) mild to moderate and (b) severe dry eye disease. There is an enlarged area of staining in (b) compared with (a)

classification tests, including identification of MGD features, as well as lipid thickness and tear volume assessment, are then performed to determine whether the disease constitutes evaporative dry eye or aqueous deficient dry eye. Lastly, the severity of disease is evaluated; for this purpose, the International Workshop on Meibomian Gland Dysfunction has provided a grading system that can be used to guide management of MGD. ${ }^{33}$

\section{Role of imaging in diagnosis}

In recent years, multiple imaging modalities have been introduced to improve the diagnosis of DED and MGD. ${ }^{34}$ These modalities aim to facilitate the evaluation of the structural and dynamic properties of the tear film. In cases of DED with tear film instability, topographic systems have been used to determine changes in the edges of the mires of a Placido disc. ${ }^{35}$ Anterior segment optical coherence tomography aims to measure the height of the tear meniscus, ${ }^{36}$ while infrared meibography provides an objective evaluation of gland structures. Tear film lipid layer thickness can be measured by interferometry, which allows objective and quantitative measurement of tear film integrity. ${ }^{37}$ 


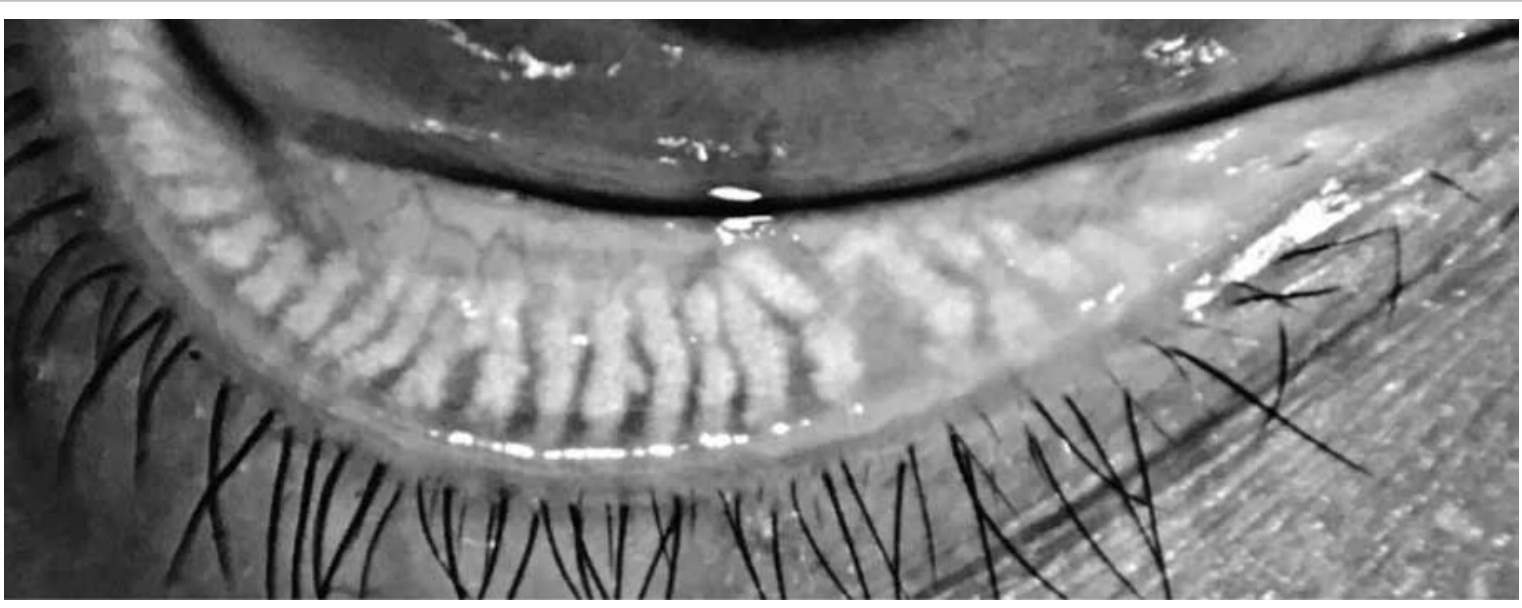

FIG 3. Normal appearance of meibomian glands under infrared illumination

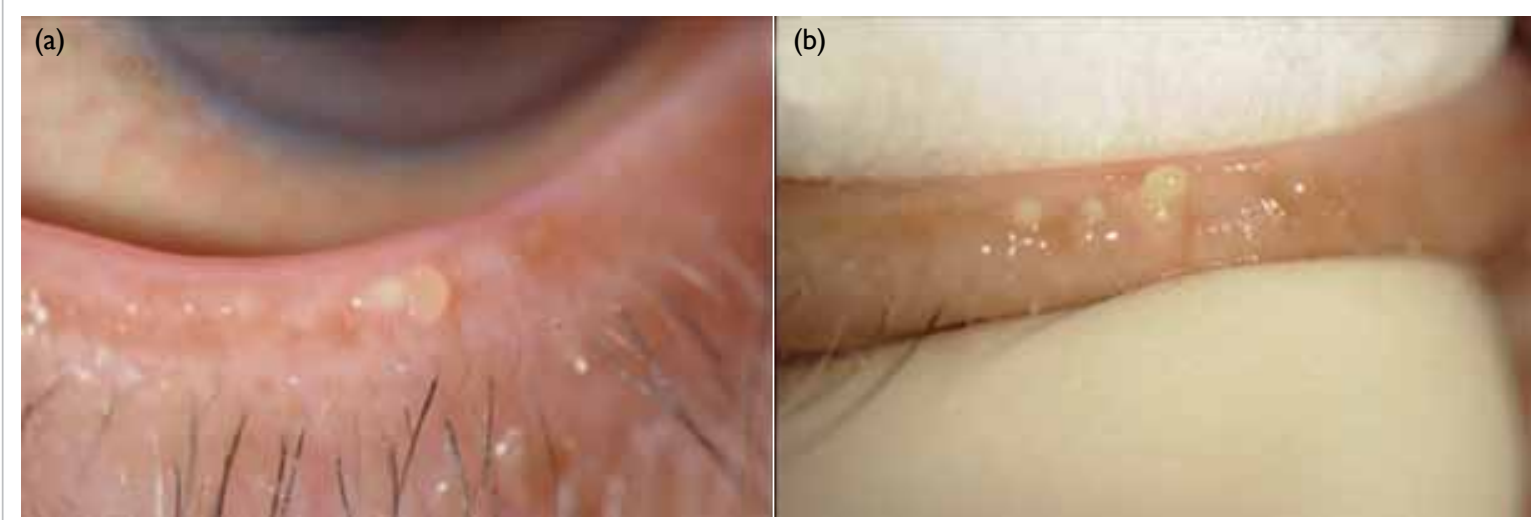

FIG 4. (a) Obstruction of a meibomian gland orifice in the lower eye lid. (b) Gentle expression of meibum from the gland to evaluate terminal duct obstruction and meibum quality

Both DED and MGD can lower the ocular surface temperature. In DED, the increased tear film evaporation rate causes heat loss, lowering ocular surface temperature. ${ }^{38}$ In MGD, lower tarsal conjunctival temperatures have been observed, increasing the viscosity of meibum; this change in viscosity leads to worsening of gland function. ${ }^{39}$ These advancements in imaging modalities have improved accuracy and standardised the diagnosis of DED and MGD.

\section{Management}

The aim of all treatment in MGD is to increase the quality and quantity of meibum expression. For this purpose, a stepwise staged approach is necessary to standardise management of the disease. ${ }^{40}$ The TFOS DEWS II created an algorithm to implement various management options, on the basis of disease severity ${ }^{40}$
Initially, patients must be educated regarding environmental and dietary modifications, which include essential fatty acid supplements. Patients must also be guided to eliminate factors contributing to the onset of DED, including contact lens wear, as well as both topical and systemic medications. Several lifestyle modifications, such as ensuring sufficient sleep or rest, maintaining appropriate hydration, and discontinuing smoking habits, may help to relieve symptoms. Ocular lubricants are suggested for mild DED; preferably, these should not contain benzalkonium chloride preservatives. Some of these modification approaches are outlined below, along with an overview of the emerging available treatment devices and options.

\section{Eyelid hygiene}

In the presence of MGD, eyelid hygiene is the 
cornerstone of MGD management. This treatment modality consists of two components: eyelid warming and eyelid massage. Meibum in patients with MGD is more stagnant and viscous and has a higher melting temperature than that in a healthy individual; thus, warming the eyelid to melt pathologically altered meibum can improve its secretion. ${ }^{33}$ Warm compression provides further benefits by melting abnormal meibum. Secretions from meibomian glands in patients with MGD exhibit lower levels of lipids, esters, and free sterols. ${ }^{41}$ Potential involvement of microbes (ie, Staphylococcus spp, Propionibacterium acnes, Bacillus oleronius, and the Demodex species described above) contributes to the pathology of MGD-associated DED by increasing meibum melting temperature and enhancing inflammation. This illustrates the importance of eyelid hygiene in MGD management. ${ }^{42}$ For patients with MGD who exhibit demodicosis, many treatment options have been described, including the use of topical $2 \%$ metronidazole. Recently, the use of tea tree oil has also increased in popularity. ${ }^{43}$ Tea tree oil is a natural essential oil that includes 4-terpineol, which is antimicrobial, anti-inflammatory, and toxic to Demodex. ${ }^{44}$ Tea tree oil lid scrubs have shown promising results as management for Demodexrelated MGD. ${ }^{45}$

Effective eyelid hygiene can be achieved by use of a hot compress (ie, soaking a clean towel in hot water, and applying the towel over the eyelids), which softens the meibum and allows better flow. After the application of the hot compress, lipid by-products can be removed gently by scrubbing both upper and lower lid margins via mild upward or downward compression of the eyelid, using a moist cotton bud; this compression begins from the nasal canthus and moves laterally. An additional therapeutic approach involves the use of mildly diluted baby shampoo for lid scrubs; this is a widely accepted therapy. Although eyelid warming and eyelid massage are efficacious for the management of MGD, they are often time-consuming and labour-intensive; thus, they encounter patient compliance issues. ${ }^{46}$ There are now a wide variety of lid cleansing products, which facilitate standardisation and simplification of treatment. Additional treatment options include warming of the lids and expression of meibomian glands, either manually (similar to above) or with the use of specially designed devices. One such device, LipiFlow (TearScience; Morrisville [NC], United States), is designed to transfer heat through the eyelid tissue to facilitate emptying of gland contents at a therapeutic temperature of $42.5^{\circ} \mathrm{C} .{ }^{47}$ Lipiflow treatment has shown promising results, and may significantly improve symptoms. ${ }^{47}$

Intense pulsed light was first reported approximately 10 years ago for the treatment of MGD, and it has demonstrated an ability to improve tear film quality and quantity, as well as to promote reduction of dry eye symptoms. ${ }^{48}$ Intraductal meibomian gland probing provides another approach to remove abnormal meibum secretions. ${ }^{49}$ Oral tetracycline and macrolides are reportedly useful in the treatment of MGD-related DED. ${ }^{40}$ These compounds are used with the assumption that inhibition of lipase production results in reduction of lipid breakdown, which may contribute to improvement in MGD. Macrolides, azithromycin in particular, exhibit anti-inflammatory properties; moreover, these compounds increase cellular accumulation of cholesterol, which may promote a suitable outcome in patients with MGDrelated DED..$^{50}$

\section{Lipid-containing artificial lubricants}

The majority of artificial tears are aqueous-based; however, these offer limited and short-term symptomatic relief, partly due to the lack of a lipid component. These artificial tears evaporate at a similar rate to that of natural tears. ${ }^{51}$ Addition of a lipid component to the artificial lubricant helps to replenish the lipid layer of the normal tear film. ${ }^{33}$ These lipid-containing lubricants exhibit long retention times and can stabilise the tear film lipid layer, reduce tear evaporation, and improve the signs of MGD. ${ }^{52}$ Additionally, lipid-containing lubricants have a longer-lasting effect and cause minimal interference of patient vision. Commercially available lipid-containing lubricants include mineral oil, highpurity castor oil, mixtures of light and standard mineral oil, and mixtures of polar phospholipid surfactant and mineral oil. A systematic review found that these lipid-containing eye drops are efficacious and safe alternatives to conventional tear lubricants in their abilities to relieve the signs and symptoms of DED. ${ }^{52}$

\section{Anti-inflammatory medications}

Because ocular surface inflammation plays an important role in the development of DED, antiinflammatory mechanisms must be considered. For patients with moderate to severe DED, low-dose topical steroids have been advocated as a treatment choice, likely because the anti-inflammatory properties of this type of drug can improve ocular inflammation through suppression of inflammatory cytokines. $^{53}$ Other anti-inflammatory options include non-glucocorticoid immunomodulatory drugs, such as topical cyclosporine A, which is an immunomodulatory drug that can reduce the expression of inflammatory markers. ${ }^{54}$ Notably, topical cyclosporine A has been proven efficacious in the treatment of DED. ${ }^{55} \mathrm{~A}$ randomised trial showed that cyclosporine A is beneficial in the stabilisation of tear film in patients with MGD. ${ }^{56}$ However, its anti-inflammatory effect is not as remarkable as that observed in DED, because the main pathophysiology 
(epithelial gland hyperkeratinisation) is not clearly resolved.

In severe cases of DED, autologous serum eye drops can be considered. Autologous serum, which is the fluid component of a patient's own blood that remains after centrifugation, exhibits similar biochemical properties to those of tears. ${ }^{40}$ Autologous serum reportedly contains specific factors that enhance epithelial regeneration, and can inhibit the release of inflammatory cytokines. ${ }^{57}$ Another treatment option for patients with severe DED involves scleral contact lenses, which are rigid gas permeable lenses of large diameter that are supported by the sclera and serve as a bridge over the corneoscleral junction. A tear reservoir is maintained between the posterior surface of the scleral contact lens and the anterior corneal surface, improving tear osmolarity and relieving dry eye symptoms. ${ }^{58}$

\section{Omega-3 dietary supplementation}

Essential fatty acid supplementation has been proven beneficial in the treatment of DED and MGD, especially when administered by intake of foods rich in omega-3 fatty acids, such as flaxseed and fish oils. ${ }^{59}$ There is a speculative association between essential fatty acids and modifications in lipid profile, as well as reductions in the fatty acid content of meibomian gland secretions. In a randomised, placebo-controlled, masked trial, omega-3 fatty acid supplementation resulted in improving ocular surface disease index score, tear breakup time, and meibum score in patients with MGD. ${ }^{60}$ Essential fatty acids also enhance the lipid layer, slow tear evaporation, and reduce apoptosis of lacrimal gland cells. ${ }^{61}$ Essential fatty acids have been reported to exhibit anti-inflammatory properties, particularly by promoting the production of prostaglandin. ${ }^{62}$ These modifications improve the tear secretion rate and tear content. Further research is needed to enhance our understanding of the underlying mechanism by which fatty acid supplementation supports the management of MGD. ${ }^{63,64}$

\section{Surgical and mechanical treatment options}

In cases where medical treatment is insufficient, surgical and mechanical treatment options include tear conservation via punctual occlusion or moisture chamber goggles. Punctal plugs retain tears on the ocular surface by blocking lacrimal drainage through the puncta (Fig 5). Permanent surgical closure may be useful when patients cannot tolerate punctual plugs. Surgical punctual occlusion blocks tear drainage and improves tear retention, and can be performed by cauterisation ${ }^{65}$ or lacrimal canalicular ligation. ${ }^{66}$ A systematic review showed that, when combined with other treatment for DED, punctual occlusion improves dry eye symptoms. ${ }^{67}$ A less invasive option, moisture chamber goggles provide a humid environment and minimise airflow to the ocular surface, thereby slowing the evaporation of tears. ${ }^{40}$

Severe DED can lead to corneal erosion, persistent epithelial defects, corneal ulceration, and eventual corneal scarring. Amniotic membrane transplant is a reasonable option in such cases. Amniotic membrane has been shown to contain multiple neurotransmitters and neurotrophic factors, which are beneficial for the management of severe DED. ${ }^{68}$ For patients with severe DED with persistent epithelial defects that are refractive to medical treatment, tarsorrhaphy may be useful. Notably, tarsorrhaphy is a procedure that achieves partial or total closure of the eyelids, either temporarily or permanently. By reducing ocular surface exposure, the rate of tear evaporation decreases, such that DED can improve. Due to unfavourable aesthetic outcomes, this approach is typically one of the final methods used for management of severe DED.

\section{Suggested treatment guideline for dry eye disease or meibomian gland dysfunction for non- ophthalmologists}

Dry eye disease and MGD are two of the most common ocular conditions encountered by medical practitioners. To manage these conditions, risk factors must be identified and modified. Notably, several environmental and lifestyle modifications can help alleviate these conditions. Proper lighting, anti-glare filters, ergonomic positioning of computer monitors, and regular break time from work may

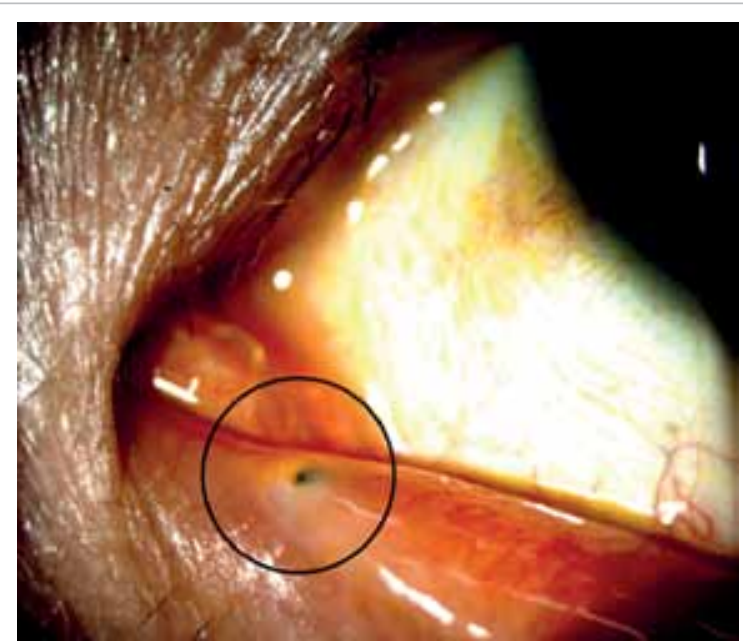

FIG 5. Appearance of punctal plug (black dot in the circle) after insertion into the lower lacrimal canalicular drainage system 
help improve the symptoms. ${ }^{69} \mathrm{~A}$ modest increase in relative humidity, achieved by using a desktoppowered humidifier, has been shown to increase subjective comfort. ${ }^{70}$ Reduction or discontinuation of contact lens use, as well as enhancement of moisture within the surrounding environment, are possible risk factor modifications. Smoking cessation can also improve the ocular surface condition and tear function. ${ }^{71}$ Lubricants can be prescribed to be used as needed for symptomatic relief. If symptoms do not resolve, benzalkonium chloride-free lubricants should be considered. Low-dose topical steroids should be implemented with particular caution, owing to the risk of steroidrelated complications (eg, cataract, glaucoma, and infection). In patients with recalcitrant disease, referral to an ophthalmologist is necessary to ensure regular monitoring. In the presence of MGD-related symptoms, lid hygiene and warm compression are strongly suggested for symptomatic control; careful manual expression of meibum should also be performed. If the above measures fail, or if the DED is secondary to other causes of aqueous deficiency (eg, Sjögren's syndrome; graft versus host disease; or chronic inflammation in Stevens-Johnson's disease, toxic epidermal necrolysis, or ocular cicatricial pemphigoid), referral to an ophthalmologist is warranted for further workup (eg, anti-SSA/Ro blood test for Sjögren's syndrome) and management.

\section{Conclusion}

Dry eye disease is a common ophthalmic problem, with a cause that is often multifactorial. Meibomian gland dysfunction is an important contributor to DED, owing to an imbalance in lipid secretion that affects the rate of tear evaporation. When tears evaporate quickly, tear osmolarity increases, resulting in DED. There are many risk factors that contribute to onset of both DED and MGD, many of which may overlap between these diseases. A clear diagnosis is vital when managing DED. Various treatment options are available for DED and MGD, and a stepwise staged approach is often crucial for ensuring appropriate management.

\section{Author contributions}

All authors contributed to the concept and design, acquisition of data, analysis and interpretation of data, drafting of the manuscript, and critical revision for important intellectual content. All authors had full access to the data, contributed to the study, approved the final version for publication, and take responsibility for its accuracy and integrity.

\section{Conflicts of interest}

As an epidemiology advisor of the Editorial Board, HKL Yuen was not involved in the peer review process of the article. All authors have disclosed no conflicts of interest.

\section{References}

1. Craig JP, Nichols KK, Akpek EK, et al. TFOS DEWS II definition and classification report. Ocul Surf 2017;15:27683.

2. The definition and classification of dry eye disease: report of the Definition and Classification Subcommittee of the International Dry Eye WorkShop (2007). Ocul Surf 2007;5:75-92

3. Tsubota K, Yokoi N, Shimazaki J, et al. New perspectives on dry eye definition and diagnosis: a consensus report by the Asia Dry Eye Society. Ocul Surf 2017;15:65-76.

4. Stapleton F, Alves M, Bunya VY, et al. TFOS DEWS II epidemiology report. Ocul Surf 2017;15:334-65.

5. Schaumberg DA, Nichols JJ, Papas EB, Tong L, Uchino M, Nichols KK. The International Workshop on Meibomian Gland Dysfunction: report of the subcommittee on the epidemiology of, and associated risk factors for, MGD. Invest Ophthalmol Vis Sci 2011;52:1994-2005.

6. The epidemiology of dry eye disease: report of the Epidemiology Subcommittee of the International Dry Eye WorkShop (2007). Ocular Surf 2007;5:93-107.

7. Nichols KK, Foulks GN, Bron AJ, et al. The International Workshop on Meibomian Gland Dysfunction: executive summary. Invest Ophthalmol Vis Sci 2011;52:1922-9.

8. Jie Y, Xu L, Wu YY, Jonas JB. Prevalence of dry eye among adult Chinese in the Beijing Eye Study. Eye (Lond) 2009;23:688-93.

9. McCarty CA, Bansal AK, Livingston PM, Stanislavsky YL, Taylor HR. The epidemiology of dry eye in Melbourne, Australia. Ophthalmology 1998;105:1114-9.

10. Bron AJ, de Paiva CS, Chauhan SK, et al. TFOS DEWS II pathophysiology report. Ocul Surf 2017;15:438-510.

11. Chhadva P, Goldhardt R, Galor A. Meibomian gland disease: the role of gland dysfunction in dry eye disease. Ophthalmology 2017;124:S20-6.

12. Gutgesell VJ, Stern GA, Hood CI. Histopathology of meibomian gland dysfunction. Am J Ophthalmol 1982;94:383-7.

13. Goto E, Endo K, Suzuki A, Fujikura Y, Matsumoto Y, Tsubota K. Tear evaporation dynamics in normal subjects and subjects with obstructive meibomian gland dysfunction. Invest Ophthalmol Vis Sci 2003;44:533-9.

14. Sullivan DA, Rocha EM, Aragona P, et al. TFOS DEWS II sex, gender, and hormones report. Ocul Surf 2017;15:283333.

15. Bron AJ, Tiffany JM. The contribution of meibomian disease to dry eye. Ocul Surf 2004;2:149-65.

16. Krenzer KL, Dana MR, Ullman MD, et al. Effect of androgen deficiency on the human meibomian gland and ocular surface. J Clin Endocrinol Metab 2000;85:4874-82.

17. Wan KH, Chen LJ, Young AL. Depression and anxiety in dry eye disease: a systematic review and meta-analysis. Eye (Lond) 2016;30:1558-67.

18. Pisella PJ, Pouliquen P, Baudouin C. Prevalence of ocular symptoms and signs with preserved and preservative free glaucoma medication. Br J Ophthalmol 2002;86:418-23.

19. Labbé A, Terry O, Brasnu E, Van Went C, Baudouin C. Tear film osmolarity in patients treated for glaucoma or ocular hypertension. Cornea 2012;31:994-9.

20. Agnifili L, Fasanella V, Costagliola C, et al. In vivo confocal microscopy of meibomian glands in glaucoma. $\mathrm{Br} \mathrm{J}$ Ophthalmol 2013;97:343-9.

21. Doughty MJ, Fonn D, Richter D, Simpson T, Caffery B, 
Gordon K. A patient questionnaire approach to estimating the prevalence of dry eye symptoms in patients presenting to optometric practices across Canada. Optom Vis Sci 1997;74:624-31.

22. Yokoi N, Yamada H, Mizukusa Y, et al. Rheology of tear film lipid layer spread in normal and aqueous tear-deficient dry eyes. Invest Ophthalmol Vis Sci 2008;49:5319-24.

23. Arita R, Itoh $\mathrm{K}$, Inoue $\mathrm{K}$, Kuchiba $\mathrm{A}$, Yamaguchi $\mathrm{T}$, Amano S. Contact lens wear is associated with decrease of meibomian glands. Ophthalmology 2009;116:379-84.

24. Henriquez AS, Korb DR. Meibomian glands and contact lens wear. Br J Ophthalmol 1981;65:108-11.

25. Alghamdi WM, Markoulli M, Holden BA, Papas EB. Impact of duration of contact lens wear on the structure and function of the meibomian glands. Ophthalmic Physiol Opt 2016;36:120-31.

26. Nettune GR, Pflugfelder SC. Post-LASIK tear dysfunction and dysesthesia. Ocul Surf 2010;8:135-45.

27. Albietz JM, Lenton LM. Management of the ocular surface and tear film before, during, and after laser in situ keratomileusis. J Refract Surg 2004;20:62-71.

28. English FP, Nutting WB. Demodicosis of ophthalmic concern. Am J Ophthalmol 1981;91:362-72.

29. Liang L, Liu Y, Ding X, Ke H, Chen C, Tseng SC. Significant correlation between meibomian gland dysfunction and keratitis in young patients with Demodex brevis infestation. Br J Ophthalmol 2018;102:1098-102.

30. Randon M, Liang $H$, El Hamdaoui $M$, et al. In vivo confocal microscopy as a novel and reliable tool for the diagnosis of Demodex eyelid infestation. Br J Ophthalmol 2015;99:33641.

31. Wolffsohn JS, Arita R, Chalmers R, et al. TFOS DEWS II diagnostic methodology report. Ocul Surf 2017;15:539-74.

32. Lemp MA, Bron AJ, Baudouin C, et al. Tear osmolarity in the diagnosis and management of dry eye disease. Am J Ophthalmol 2011;151:792-8.e1.

33. Geerling G, Tauber J, Baudouin C, et al. The International Workshop on Meibomian Gland Dysfunction: report of the subcommittee on management and treatment of meibomian gland dysfunction. Invest Ophthalmol Vis Sci 2011;52:2050-64.

34. Chan TC, Wan KH, Shih KC, Jhanji V. Advances in dry eye imaging: the present and beyond. $\mathrm{Br} \mathrm{J}$ Ophthalmol 2018;102:295-301.

35. Goto T, Zheng X, Okamoto S, Ohashi Y. Tear film stability analysis system: introducing a new application for videokeratography. Cornea 2004;23:S65-70.

36. Ibrahim OM, Dogru M, Takano Y, et al. Application of visante optical coherence tomography tear meniscus height measurement in the diagnosis of dry eye disease. Ophthalmology 2010;117:1923-9.

37. Finis D, Pischel N, Schrader S, Geerling G. Evaluation of lipid layer thickness measurement of the tear film as a diagnostic tool for meibomian gland dysfunction. Cornea 2013;32:1549-53.

38. Purslow C, Wolffsohn J. The relation between physical properties of the anterior eye and ocular surface temperature. Optom Vis Sci 2007;84:197-201.

39. Arita R, Shirakawa R, Maeda S, Yamaguchi M, Ohashi Y, Amano S. Decreased surface temperature of tarsal conjunctiva in patients with meibomian gland dysfunction. JAMA Ophthalmol 2013;131:818-9.

40. Jones L, Downie LE, Korb D, et al. TFOS DEWS II management and therapy report. Ocul Surf 2017;15:575628.

41. Shine WE, McCulley JP. Polar lipids in human meibomian gland secretions. Curr Eye Res 2003;26:89-94.

42. Knop E, Knop N, Millar T, Obata H, Sullivan DA. The International Workshop on Meibomian Gland Dysfunction: report of the subcommittee on anatomy, physiology, and pathophysiology of the meibomian gland. Invest Ophthalmol Vis Sci 2011;52:1938-78.

43. Gao YY, Di Pascuale MA, Elizondo A, Tseng SC. Clinical treatment of ocular demodecosis by lid scrub with tea tree oil. Cornea 2007;26:136-43.

44. Tighe S, Gao YY, Tseng SC. Terpinen-4-ol is the most active ingredient of tea tree oil to kill Demodex mites. Transl Vis Sci Technol 2013;2:2.

45. Gao YY, Xu DL, Huang IJ, Wang R, Tseng SC. Treatment of ocular itching associated with ocular demodicosis by $5 \%$ tea tree oil ointment. Cornea 2012;31:14-7.

46. Korb DR, Blackie CA. Meibomian gland therapeutic expression: quantifying the applied pressure and the limitation of resulting pain. Eye Contact Lens 2011;37:298301.

47. Lane SS, DuBiner HB, Epstein RJ, et al. A new system, the LipiFlow, for the treatment of meibomian gland dysfunction. Cornea 2012;31:396-404.

48. Craig JP, Chen YH, Turnbull PR. Prospective trial of intense pulsed light for the treatment of meibomian gland dysfunction. Invest Ophthalmol Vis Sci 2015;56:1965-70.

49. Sik Sarman Z, Cucen B, Yuksel N, Cengiz A, Caglar Y. Effectiveness of intraductal meibomian gland probing for obstructive meibomian gland dysfunction. Cornea 2016;35:721-4.

50. Liu Y, Kam WR, Ding J, Sullivan DA. Can tetracycline antibiotics duplicate the ability of azithromycin to stimulate human meibomian gland epithelial cell differentiation? Cornea 2015;34:342-6.

51. Trees GR, Tomlinson A. Effect of artificial tear solutions and saline on tear film evaporation. Optom Vis Sci 1990;67:886-90.

52. Lee SY, Tong L. Lipid-containing lubricants for dry eye: a systematic review. Optom Vis Sci 2012;89:1654-61.

53. Djalilian AR, Nagineni CN, Mahesh SP, Smith JA, Nussenblatt RB, Hooks JJ. Inhibition of inflammatory cytokine production in human corneal cells by dexamethasone, but not cyclosporin. Cornea 2006;25:70914.

54. Gao J, Sana R, Calder V, et al. Mitochondrial permeability transition pore in inflammatory apoptosis of human conjunctival epithelial cells and T cells: effect of cyclosporin A. Invest Ophthalmol Vis Sci 2013;54:4717-33.

55. Wan KH, Chen LJ, Young AL. Efficacy and safety of topical $0.05 \%$ cyclosporine eye drops in the treatment of dry eye syndrome: a systematic review and meta-analysis. Ocul Surf 2015;13:213-25.

56. Prabhasawat P, Tesavibul N, Mahawong W. A randomized double-masked study of $0.05 \%$ cyclosporine ophthalmic emulsion in the treatment of meibomian gland dysfunction. Cornea 2012;31:1386-93.

57. López-García JS, García-Lozano I, Rivas L, Giménez C, Acera A, Suárez-Cortés T. Effects of autologous serum eye drops on conjunctival expression of $M U C 5 A C$ in patients with ocular surface disorders. Cornea 2016;35:336-41.

58. La Porta Weber S, Becco de Souza R, Gomes JÁ, Hofling- 
Lima AL. The use of the Esclera scleral contact lens in the treatment of moderate to severe dry eye disease. Am J Ophthalmol 2016;163:167-73.e1.

59. Liu Y, Kam WR, Sullivan DA. Influence of omega 3 and 6 fatty acids on human meibomian gland epithelial cells. Cornea 2016;35:1122-6.

60. Macsai MS. The role of omega-3 dietary supplementation in blepharitis and meibomian gland dysfunction (an AOS thesis). Trans Am Ophthalmol Soc 2008;106:336-56.

61. Rosenberg ES, Asbell PA. Essential fatty acids in the treatment of dry eye. Ocul Surf 2010;8:18-28.

62. Das UN. Essential fatty acids-a review. Curr Pharm Biotechnol 2006;7:467-82.

63. Barabino S, Rolando M, Camicione P, et al. Systemic linoleic and gamma-linolenic acid therapy in dry eye syndrome with an inflammatory component. Cornea 2003;22:97-101.

64. Aragona P, Bucolo C, Spinella R, Giuffrida S, Ferreri G. Systemic omega-6 essential fatty acid treatment and pge1 tear content in Sjögren's syndrome patients. Invest Ophthalmol Vis Sci 2005;46:4474-9.

65. Ohba E, Dogru M, Hosaka E, et al. Surgical punctal occlusion with a high heat-energy releasing cautery device for severe dry eye with recurrent punctal plug extrusion. Am J Ophthalmol 2011;151:483-7.e1.

66. DeMartelaere SL, Blaydon SM, Tovilla-Canales JL, Shore JW. A permanent and reversible procedure to block tear drainage for the treatment of dry eye. Ophthalmic Plast Reconstr Surg 2006;22:352-5.

67. Ervin AM, Law A, Pucker AD. Punctal occlusion for dry eye syndrome. Cochrane Database Syst Rev 2017;(6):CD006775.

68. Sakuragawa N, Elwan MA, Uchida S, Fujii T, Kawashima K. Non-neuronal neurotransmitters and neurotrophic factors in amniotic epithelial cells: expression and function in humans and monkey. Jpn J Pharmacol 2001;85:20-3.

69. Blehm C, Vishnu S, Khattak A, Mitra S, Yee RW. Computer vision syndrome: a review. Surv Ophthalmol 2005;50:25362.

70. Wang MT, Chan E, Ea L, et al. Randomized trial of desktop humidifier for dry eye relief in computer users. Optom Vis Sci 2017;94:1052-7.

71. Aktaş S, Tetikoğlu M, Koçak A, et al. Impact of smoking on the ocular surface, tear function, and tear osmolarity. Curr Eye Res 2017;42:1585-9. 